\title{
The Influence of Pyrazinamide Monoresistance on Treatment Outcomes in Tuberculosis Patients from Southern China
}

\author{
Shouyong Tan ${ }^{*}$, Yunwei Rao ${ }^{2}$, Jintao Guo ${ }^{3}$, Yaoju Tan${ }^{1}$, Xingshan Cai1, Haobin Kuang1, \\ Yanqiong Li' ${ }^{1}$, Wenyan Liu', Julius Mugweru ${ }^{3}$, Bangxing Wang', Yuanyuan $\mathrm{CaO}^{3}$, \\ Changwei Wang ${ }^{3}$, Ying Zhang ${ }^{4}$, Tianyu Zhang ${ }^{*}$ \\ ${ }^{1}$ State Key Laboratory of Respiratory Disease, The Guangzhou Chest Hospital, Guangzhou, China \\ ${ }^{2}$ Respiratory Department, Guangzhou Medical University, Guangzhou, China \\ ${ }^{3}$ State Key Laboratory of Respiratory Disease, Guangzhou Institutes of Biomedicine and Health, Chinese \\ Academy of Sciences, Guangzhou, China \\ ${ }^{4}$ W. Harry Feinstone Department of Molecular Microbiology and Immunology, Bloomberg School of Public \\ Health, Johns Hopkins University, Baltimore, USA \\ Email: "tanshyo@163.com, "zhang_tianyu@gibh.ac.cn
}

Received 11 December 2015; accepted 6 March 2016; published 9 March 2016

Copyright @ 2016 by authors and Scientific Research Publishing Inc.

This work is licensed under the Creative Commons Attribution International License (CC BY).

http://creativecommons.org/licenses/by/4.0/

(c) (i) Open Access

\section{Abstract}

This study aimed to explore the influence of pyrazinamide (PZA) monoresistance on the treatment outcome of otherwise drug susceptible tuberculosis (TB). A cohort of 194 TB patients that were infected with strains susceptible to isoniazid (INH), rifampin (RIF) and ethambutol (EMB) were included in a retrospective study at the Guangzhou Chest Hospital. We reported 148 (76.3\%) PZAsusceptible TB cases and $46(23.7 \%)$ PZA-monoresistance TB cases identified by the BACTEC MGIT 960 system. All patients were treated with the standard 6 months WHO recommended regimen, which included 2 months of INH + RIF + EMB + PZA in the intensive-phase, and the subsequent 4 months of INH + RIF during continuation-phase. Bacterial burden in the lungs was estimated using sputum smear acid-fast bacillary count while the lung lesions and cavitations were examined by $\mathrm{X}$-ray at the end of first 2 months of chemotherapy. After intensive-phase treatment, there were $164(84.5 \%)$ cases of smear-negative conversion and $151(77.9 \%)$ cases of total or partial lesion elimination. The rates of smear-negative conversion (78.3\%) and lesion elimination $(39.1 \%)$ of the PZA-monoresistant patients were similar with the PZA-sensitive group $(P>0.05)$. However, lung cavitation was more likely to be resolved in PZA-sensitive patients than in the PZA-patients $\left(\mathrm{X}^{2}=9.623, \mathrm{P}=0.002\right)$. The smear-negative conversion rates were $95.9 \%$ for the PZA-sensitive patients and $87.0 \%$ for the PZA-monoresistant patients after 6 months of treatment $\left(\mathrm{X}^{2}=3.461, \mathrm{P}=\right.$

\footnotetext{
${ }^{*}$ Corresponding authors.
}

How to cite this paper: Tan, S.Y., et al. (2016) The Influence of Pyrazinamide Monoresistance on Treatment Outcomes in Tuberculosis Patients from Southern China. Journal of Tuberculosis Research, 4, 9-17. 
0.063). Together, our data suggest that PZA-monoresistance contributes to the delay of resolution of the lung cavitations in the Southern China population without affecting the sputum conversion and lesion elimination rates.

\section{Keywords}

\section{Tuberculosis, Pyrazinamide, Treatment, Monoresistance}

\section{Introduction}

Pyrazinamide (PZA) is a unique first-line anti-TB drug that acts against the tubercle bacilli at the acidic environment ( $\mathrm{pH}<5.5)$ of caseous lesions caused by inflammation [1]. Though PZA has no bactericidal activity against the actively growing bacilli, it has sterilizing activity against the semi-dormant tubercle bacilli or persisters [2] [3]. Therefore, PZA is recommended by the World Health Organization (WHO) as an important component of the directly observed treatment short course (DOTS) strategy against TB. Several studies reported bactericidal effect of PZA in the first two months of treatment, which may be related to the acid environment created by active inflammatory lesions [2] [4]. Inclusion of PZA in the first two months of treatment therefore can shorten the overall length of treatment to 6 months despite the fact that the bactericidal effect was not seen when PZA is used alone [5].

Pre-existing drug resistance against the conventional first-line anti-tuberculosis drugs in standardized treatment strategy may increase the risk of acquiring resistance to the new drugs used in combinations [6]. The standardized regimen for initial TB treatment uses combination of isoniazid (INH), rifampin (RIF), PZA and ethambutol (EMB) in the intensive phase for 2 - 4 months, followed by a 4-month continuation phase treatment with INH and RIF. However, whether the PZA-monoresistance can affect the therapeutic outcomes is unclear. In this study we explored the effect of PZA-monoresistance on TB treatment outcome in otherwise drug susceptible TB patients.

\section{Materials and Methods}

\subsection{Study Population}

This was a retrospective cohort study at the Guangzhou Chest Hospital between January 2011 and December 2013. Together, 194 patients who met the selection criteria of positive pulmonary tuberculosis and were susceptible to INH, RIF and EMB were enrolled (Table 1). There were 124 (63.9\%) male and $70(36.1 \%)$ female patients (Table 2), gender ratio between male and female was 1.77:1. Patients were aged between 15 to 82 years (mean age 38.1 years), and 45 (23.2\%) cases had history of TB.

\subsection{Drug Treatment}

Patients were treated for 6 months with WHO recommended intensive-phase regimen of INH + RIF + PZA + EMB for the first 2 months and continuation-phase regimen of INH + RIF for additional 4 months. Dosages of INH, RIF, PZA and EMB were in accordance with published guidelines [7]. RIF was administered on empty stomach. All drugs used in this study were purchased from licensed suppliers: INH from Yichang Humanwell Pharmaceutical CO., Ltd (Hubei, China), RIF from Southern China Pharmaceutical CO., Ltd (Guangdong, China), EMB from Chengdu Jinhua Pharmaceutical CO., Ltd (Sichuan, China) and PZA from Guangdong Taicheng Pharmaceutical CO., Ltd. (Guangdong, China).

\subsection{Sputum Collection}

Early morning sputum specimens were collected for smear testing and liquid culture detection. Baseline sputum specimens were collected from all qualified patients at the date of their clinical enrollment. Another two sputum samples were collected after completion of intensive phase treatment (1 sputum sample on the day of completion and 1 sputum sample prior to the second dose of continuation-phase treatment). Unless otherwise specified, 
Table 1. Patient selection criteria.

1) Patients who met the criteria of TB diagnosis and treatment guidelines made by TB Branch of Chinese Medical Association [39];

2) Mtb strains from them were sensitive to INH + RIF + EMB according to pre-treatment drug susceptibility testing;

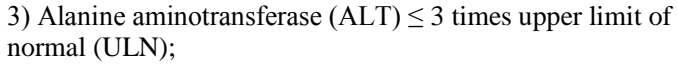

4) Total bilirubin $\leq 2.5$ times ULN;

5) Creatinine $\leq 2$ times ULN;

6) Hemoglobin $\geq 7.0 \mathrm{~g} / \mathrm{dL}$;

7) Platelets $\geq 100,000 / \mathrm{mm}^{3}$;

8) Non-pregnant;

9) $\leq 5$ days of anti-tuberculosis treatment in last 6 months.
1) Patients with severe liver and kidney disease history;

2) Mental illness, gout and diabetes history;

3) HIV antibody positive, long-term use of immunosuppressive agents, severe malnutrition;

4) Currently enrolled in or was going to start medications of antiretroviral and/or immunosuppressive agents in next 8 weeks.

5) Discontinuation of treatment due to serious adverse effects;

6) Failure to complete the six-month treatment program;

7) Pregnancy or breast-feeding;

8) Weight $<40 \mathrm{~kg}$;

9) Central nervous system tuberculosis;

10) Pulmonary silicosis.

sputum samples were collected on a monthly basis during the continuation-phase treatment. Sputum samples were digested and de-contaminated using conventional $\mathrm{N}$-acetyl-L-cysteine-NaOH (NALC-NaOH) method [8] with final concentrations of $1 \%-2 \%$ for $\mathrm{NaOH}$ and $0.25 \%$ for NALC. Sediments were used for smear and culture.

\subsection{Acid-Fast Bacilli (AFB) Smear}

AFB smear procedures for the sputum samples were adopted from the Chinese Anti-Tuberculosis Association [9]. Briefly, thin smears were prepared from the sputum sediments and were stained using the Nepal luxuriant staining. The smears were examined by hospital technicians under optical microscopes at $\times 100$ oil immersion magnification. Number of AFB was estimated per 100 microscopic fields. Smear was reported negative when no AFB was detected from at least 100 microscopic fields per sample. A score was given to the AFB positive sample according to the WHO guideline [10]: sample with 10 to 99 AFB observed per 100 fields scored as $1+; 1$ to 10 AFB per field in at least 50 fields was scored 2+; sample was scored $3+$ when $\geq 10$ AFB observed per field in at least 20 fields. Patients were reported as smear-negative when they maintained sputum AFB negative for 3 consecutive months.

\subsection{Mycobacterial Culture and Drug Susceptibility Test Using MGIT 960 Liquid Culture System}

An antibiotic mixture of polymyxin B, amphotericin B, nalidixic acid, trimethoprim, and azlocillin (PANTA) was reconstituted with $15.0 \mathrm{ml}$ MGIT growth supplement and $0.8 \mathrm{ml}$ PANTA was added to each MGIT tube prior to sample inoculation. Sputum sediments were diluted 1:5 with PBS and a $0.5 \mathrm{ml}$ inoculums was added to the MGIT tube. Samples were incubated in a BACTEC MGIT 960 Mycobacterium Detection System (Becton Dickinson, Sparks, MD) at $37^{\circ} \mathrm{C}$ according to the manufacturer's instructions. Fluorescence was recorded automatically by a 365-nm UV transilluminator. Time of incubation for mycobacterium detection was based on the date of the earliest instrument positivity. The result was confirmed by the AFB smear positivity.

Drug susceptibility of the clinical isolates was performed as described [11] [12] prior to the initiation of drug treatment. The three first-line anti-tuberculosis drugs with final concentration of INH $0.1 \mu \mathrm{g} / \mathrm{ml}, \mathrm{RIF} 1 \mu \mathrm{g} / \mathrm{ml}$ and EMB $3.5 \mu \mathrm{g} / \mathrm{ml}$ were prepared and added to the MGIT tubes enriched with PANTA respectively according to the manufacturer's instructions. A growth control tube was prepared without the three first-line anti-tuberculosis drugs. The PZA susceptibility test was performed using the BACTEC MGIT 960 PZA Kit (Becton Dick- 
inson, Sparks, MD). Isolates were tested against PZA in $100 \mu \mathrm{g} / \mathrm{ml}$ and compared with a growth control at a reduced $\mathrm{pH}$ of 5.9. The tubes were incubated with $0.5 \mathrm{ml}$ inoculums at $37^{\circ} \mathrm{C}$ in the BACTEC MGIT 960 instrument. Tube inoculated with Mtb strain H37Rv (ATCC 27294) was used as a drug-susceptible (negative) control. The tubes were monitored between the 4th to the 21st day post-incubation. On the day the growth control tube showed positive growth, the sputum from the drug-containing samples were compared to their growth controls and to the Mtb H37Rv. Data of individual anti-tuberculosis drug tested was interpreted and reported automatically by the instrument. Susceptibility profile was determined when the growth unit (GU) of the growth control reached 400, a drug-treated sample with $\mathrm{GU} \geq 100$ was defined as drug-resistant (R) and sample with $\mathrm{GU} \leq 100$ was considered susceptible (S). Susceptibility to EMB and PZA was cross-compared with Mtb H37Rv. The patients were categorized into PZA-sensitive and PZA-monoresistant groups with respect to their PZA susceptibility profile before and after treatment.

\subsection{Lung Lesion and Cavity Examination}

Chest X-ray scanning and computed tomography (CT) images were reviewed by two attending physicians before and after 2-month intensive-phase treatment. Conditions and sizes of the lung lesions and cavities after drug treatment were compared with that of pre-treatment phase.

\subsection{Statistics}

Data from the study was expressed as percentages and the chi-square $\left(\mathrm{X}^{2}\right)$ test was used to compare the rates between PZA-susceptible and PZA-resistant groups. If the theoretical frequency was $<5$, the continuity correction was used while a P value less than 0.05 was considered statistically significant. Analysis was carried out using SPSS (version 13.0; SPSS; Chicago, IL) statistical software.

\subsection{Ethics Approval}

This study was approved by the Ethics Committee of Guangzhou Chest Hospital. Written informed consent was given by all participants. Patient information was collected and recorded by attending physicians.

\section{Results}

Here we report 148 PZA-susceptible TB cases comprising of 100 (67.6\%) males and 48 females (gender ratio = 1.48 male: 1 female). Mean age was 42.1 years (ranged 15 to 76 years). For the PZA-monoresistance TB group, $24(52.2 \%)$ patients were males and 22 were females (gender ratio $=1.09$ male: 1 female), mean age was 36.1 years (ranged 16 to 77 years, Table 2).

Table 2 shows before the start of drug treatment, there were 69 (35.6\%) cases of low sputum smear AFB count $(+1), 61(31.4 \%)$ cases of AFB score $2+$ and $64(33.0 \%)$ cases were registered as high AFB count $(\geq 3+)$. About half of the enrolled patients had 5 - 6 pulmonary lesions (98 cases of 194, 50.5\%), and 163 of 194 patients (84.0\%) had cavity lesions (Table 2).

After 2 months of intensive treatment with INH + RIF + PZA + EMB, 164 of 194 (84.5\%) patients exhibited sputum smear negative conversion while 30 (15.5\%) cases remained non-conversion. Ninety-six of 194 cases (49.5\%) had total lesion elimination, 55 of 194 cases (28.4\%) showed partial lesion elimination and 43 of 194 (22.1\%) failed to resolve lung lesions after treatment (Table 3). Overall, 77.9\% (151 of 194) of TB patients had total or partial lesion elimination. 78.4\% (126 of 148 cases) PZA-sensitive patients had lesion elimination and $75.1 \%$ (35 of 46) of the PZA-monoresistance patients had lesion elimination $\left(X^{2}=0.297, P=0.537\right.$ ) (Table 3). The size of cavity lesions shrunk in 113 of 163 (69.3\%) patients who had lung cavitations at the time of enrollment. It is of interest to note that $75.4 \%$ (95 of 126 cases) PZA-sensitive patients had lung cavitations resolution after 2-month intensive treatment. In contrast, 48.6\% (18 of 37) of the PZA-monoresistance patients recovered from lung cavitations $\left(\mathrm{X}^{2}=9.623, \mathrm{P}=0.002\right)$ (Table 3 ).

On completion of the continuation-phase treatment with INH and RIF, a robust 93.8\% (182 of 194 cases) of TB patients reported sputum smear negative conversion while 12 cases (6.2\%) did not convert. The conversion rate in PZA-susceptible patients was 95.9\% (142 of 148) while 87.0\% (40 of 46 cases) of PZA-monoresistant patients had sputum smear negative conversion. Overall, there was no clear difference found between PZA-sensitive and PZA-monoresistant groups in terms of sputum smear negative conversion rate $\left(X^{2}=3.461, P=0.063\right)$. 
Table 2. Summary of patient characteristics before treatment.

\begin{tabular}{|c|c|c|c|c|c|}
\hline \multicolumn{2}{|c|}{ Characteristic } & $\begin{array}{c}\text { PZA-sensitive } \\
\text { cases (\%) }\end{array}$ & PZA-resistant & $x^{2}$ & $P$ value \\
\hline \multirow{3}{*}{ Gender } & Male & $100(67.6)$ & $24(52.2)$ & \multirow{3}{*}{3.606} & \multirow{3}{*}{0.067} \\
\hline & & & & & \\
\hline & Female & $48(32.4)$ & $22(47.8)$ & & \\
\hline \multirow{2}{*}{ Medical history } & Initial treatment ${ }^{\mathrm{a}}$ & $113(76.4)$ & $36(78.3)$ & \multirow{2}{*}{0.072} & \multirow{2}{*}{0.978} \\
\hline & Retreatment ${ }^{\mathrm{b}}$ & 35 (23.6) & $10(21.7)$ & & \\
\hline \multirow{4}{*}{ Age (years) } & $\leq 25$ & 44 (29.7) & $17(37.0)$ & \multirow{4}{*}{3.892} & \multirow{4}{*}{0.273} \\
\hline & $25-44$ & 53 (35.8) & $20(43.5)$ & & \\
\hline & $45-64$ & 35 (23.7) & $6(13.0)$ & & \\
\hline & $\geq 65$ & $16(10.8)$ & $3(6.5)$ & & \\
\hline \multirow{3}{*}{ Sputum smear } & $1+$ & $52(35.1)$ & $17(37.0)$ & \multirow{3}{*}{0.665} & \multirow{3}{*}{0.721} \\
\hline & $2+$ & 45 (30.4) & $16(34.8)$ & & \\
\hline & $\geq 3+$ & $51(34.5)$ & $13(28.2)$ & & \\
\hline \multirow{4}{*}{ Lesions of infection } & $1-2$ & 28 (18.9) & $9(19.6)$ & \multirow{3}{*}{3.907} & \multirow{3}{*}{0.142} \\
\hline & $3-4$ & $40(27.0)$ & 19 (41.3) & & \\
\hline & $5-6$ & $80(54.1)$ & $18(39.1)$ & & \\
\hline & Cavitary lesions & $126(85.1)$ & 37 (80.4) & 0.577 & 0.447 \\
\hline
\end{tabular}

${ }^{\mathrm{a}} \mathrm{A}$ patient was classified as initial-treatment when he or she had no previous anti-tuberculosis treatment; ${ }^{\mathrm{b}} \mathrm{A}$ patient was classified as re-treatment when 1) A TB patient who had completed treatment and was declared cured by a physician in the past, but who reported back to the health facility and found to be sputum smear-positive; 2) A smear-positive TB patient after regular 6 months preliminary treatment and also initial smear negative patient who turned positive again; 3) TB patients who received anti-tuberculosis treatment for one month or more from any source and returns to treatment after having defaulted, i.e., not taken anti-TB drugs consecutively for two months or more, and were found to be sputum smear-positive; 4) Chronic Mtb excreter.

Table 3. Clinical outcomes after 2 months intensive-phase treatment.

\begin{tabular}{|c|c|c|c|c|c|}
\hline \multicolumn{2}{|c|}{ Characteristic } & $\begin{array}{c}\text { PZA-sensitive } \\
\text { cases (\%) }\end{array}$ & $\begin{array}{c}\text { PZA-resistant } \\
\text { cases (\%) }\end{array}$ & $x^{2}$ & $\mathrm{P}$ value \\
\hline \multirow{3}{*}{ Sputum smear $^{*}$} & Conversion & $128(86.5)$ & 36 (78.3) & \multirow{3}{*}{1.816} & \multirow{3}{*}{0.178} \\
\hline & & & & & \\
\hline & Non-conversion & $20(13.5)$ & $10(21.7)$ & & \\
\hline \multirow{6}{*}{ Lesion elimination $^{\#}$} & Total lesion elimination & $75(50.7)$ & $21(45.7)$ & \multirow{3}{*}{0.279} & \multirow{3}{*}{0.597} \\
\hline & Partial elimination & $41(27.7)$ & $14(30.4)$ & & \\
\hline & Non-elimination & $32(21.6)$ & 11 (23.9) & & \\
\hline & Shrunk cavities & $95(75.4)$ & $18(48.7)$ & \multirow{3}{*}{9.623} & \multirow{3}{*}{0.002} \\
\hline & & & & & \\
\hline & Non-shrunk cavities & $31(24.6)$ & 19 (51.3) & & \\
\hline
\end{tabular}

* On completion of 2 months treatment, if patients had negative results in both sputum AFB smear test and liquid culture, they were considered as sputum negative conversion. Patients with negative sputum AFB smear but were positive for liquid culture after 2 months treatment were considered as sputum negative non-conversion; "\#he terms in lesion and cavity conditions were defined as follows: non-elimination, the lesions did not change obviously; partial elimination, less than one-third of lesions had decreased than in the pre-treatment phase; total lesion elimination, compared with pre-treatment, more than one-third of lesions had decreased; non-shrunk cavities, the sizes of cavities became larger or unchanged compared with pre-treatment; shrunk cavities, cavities decreased in size compared with pre-treatment. 


\section{Discussion}

In this retrospective cohort study evaluating the impact of PZA-monoresistance on treatment outcomes, no significant differences were found in terms of rates of 2- and 6-month sputum smear conversion, while reduced cavity size was more pronounced in PZA-susceptible patients compared to PZA-monoresistance patients.

The rate of smear conversion after two months of treatment is an important indicator for TB cure [13]. Singla et al. (2003), showed that a smear conversion rate of at least $75 \%$ is a good indicator for non-resistant smear positive TB [14]. In addition, non-conversion of positive smears after the first two months of treatment is one of the reliable predictors for treatment failure [15]. Several factors may delay the time to smear conversion, including lung cavitations, extensive lesions, grade of sputum smear AFB prior to treatment and age. Some studies reported that drug resistance is strongly associated with late ( $>8$ weeks) culture conversion as the failure of TB treatment [16] [17].

PZA is an important first-line drug used in the first two months of treatment to reduce the duration of treatment required [18]. A multicenter clinical study showed that combination of PZA with INH and streptomycin (STR) could reduce TB relapse rate from $29 \%$ to $8 \%$ after 6 months of treatment [4]. Though it was also suggested that PZA plays no role in combination with RIF and INH to treat Mycobacterium bovis, a strain naturally resist to PZA [19].

It has been reported that pre-existing RIF resistance will lower the curative rate and increase the rate of relapse after the 6-month treatment regimen, especially when RIF was given alone during the initial 2-month intensive phase of treatment [20]. However, the pre-existing resistance of INH or STR had little effect on the sterilizing activity of treatment regimen that included RIF or PZA as partner drug, possibly due to the more potent sterilizing activity of PZA [21]. The actual effect of PZA resistance on the treatment outcome was not well studied [22].

This study reports a smear conversion rate of 78.3\% (36 of 46 cases) for the PZA-monoresistant patients, while that of PZA-sensitive patients was slightly higher (128/148 cases, 86.5\%) after the 2 months intensivephase treatment. No significant difference $(\mathrm{P}>0.05)$ was observed between the PZA-monoresistant and PZAsensitive patients in total and partial lesion elimination rates after the intensive phase (Table 3). This is consistent with our previous study [23] and a recent report in San Francisco, California [24]. Liang et al. reported that the multiple drug resistance, but not the single drug-resistance, could alter the sputum conversion after 2 months treatment [25]. As PZA has mainly bacteriostatic activity even when used alone at high dose in mouse [26], the pre-exist PZA-resistance may not affect the culture conversion rate in patients when multiple first-line drugs were used in combination for the first 2-months of treatment [27]. The synergistic effect of the other partner drugs in treatment regimen can compensate the lost of PZA function, though RIF and PZA can contribute to stronger synergy of bactericidal activities for the first two months of treatment [27] [28]. Manca et al. reported that PZA could down-regulate the release of pro-inflammatory cytokines and chemokines, including IL- $1 \beta$, IL-6, TNF and MCP-1. Since the inflammatory cytokines and chemokines can drive the manifestations of cavitations and lung pathology [29], immunosuppression by PZA may be a promising adjunctive treatment to prevent lung pathology or cavitation despite PZA-resistance in Mtb is shown in vitro [30].

Cavity formation facilitates bacterial killing by promoting phagosome acidification, production of reactive oxygen and nitrogen species, hydrolytic enzymes, and cationic antimicrobial peptides [31] [32]. After two months of WHO recommended intensive treatment with INH + RIF + PZA + EMB, we found that the proportion of patients with cavitations resolution was significantly lower $(\mathrm{P}=0.002)$ in the PZA-monoresistant group (48.7\%, 18 of 37 cases) than that of the PZA-susceptible patients ( $75.4 \%$, 95 of 126 cases). We speculate that the acidification and hypoxic micro-environment in the caseous necrosis and lesions [25] of the PZA-sensitive patients enhances the sterilizing activity of PZA [33] [34]. Therefore it leads to better healing of cavity lesions in the PZA-susceptible group compare to that in the PZA-resistant group.

These findings reveal a slightly higher, but not statistically significant $(\mathrm{P}=0.178)$, smear conversion rate in the PZA-sensitive patients (86.5\%, 128 of 148 cases) than the PZA-resistant patients (78.3\%, 36 of 46 cases) (Table 3). Yee et al. reported a significantly worse clinical outcome in patients with PZA-monoresistance TB than patients with fully susceptible strains from a study of 218 patients in Canada [35]. The main limitation of this study was the inability to follow the patients for long-term relapses. However, our findings are consistent with previous results that the cavity improvement for PZA-sensitive patients is better than the resistant patients. This may partially explain the differential conclusion of this study that based on the cavity improvement and 
smear conversion rate after two months of intensive treatments whereas the other study measured bacteria relapse in long term [36]. It is also in agreement with another report that showed surprisingly high relapse rates in patients with either positive culture after two months of treatment or had cavities prior to treatment [36]. Accurate PZA susceptibility testing is very difficult that some strains may be falsely classified as PZA-resistant by the phenotypic test. However, our recent study indicated that the PZA susceptibility testing using MGIT 960 system was relatively reliable as all the clinical strains with pncA mutation were phenotypic PZA-resistant [37]. This is also consistent with the finding that $p n c A$ encodes the enzyme that activates the pro-drug PZA into its active form pyrazinoic acid for action [38]. Sequencing the $p n c A$ and $r p s A$ genes from the Mtb isolates may help to identify PZA-resistant strain since mutations in these genes could be related to PZA resistance. However, the mechanism of PZA resistance still need to be studied further as mutations in these 2 genes may not be responsible for PZA resistance and many clinical PZA-resistant isolates do not have known mutations in pncA and rpsA [22].

\section{Conclusion}

In summary, PZA is an important drug for TB treatment. Although there was no significant difference in smear negative conversion rates between the PZA-monoresistant and PZA-sensitive patients after two months intensive treatment, PZA-sensitive patients achieved better recovery of lung cavitation. Without a reliable PZA susceptibility testing and a definite correlation between the susceptibility of PZA tested in vitro and that in vivo, we suggest that the doctors should use PZA in patients regardless of the current PZA susceptibility testing results. However, the possibility of Mtb relapse and spreading depends on lung cavities; the delay of cavitations resolution also poses a risk of prolonging Mtb transmission. With technological advances and growing knowledge of the mechanism underlying PZA-resistance, our data suggest that personalized combinations of drugs, other than standard regimen, should be tailored to individual patients in the future with consideration of their initial PZAsusceptibility.

\section{Acknowledgements}

This work was supported by One Hundred Talents Program of the Chinese Academy of Sciences (Category A, to T.Z.), by the Key Program of the Chinese Academy of Sciences (KJZD-EW-L02), by the National Natural Science Foundation of China (81572037), by the Key Project Grant (SKLRD2016ZJ003) and the Open Project Grant (2014SKLRD-O06) from the State Key Lab of Respiratory Disease, Guangzhou Institute of Respiratory Disease, First Affiliated Hospital of Guangzhou Medical University, by the Chinese Academy of Sciences (CAS)Commonwealth Scientific and Industrial Research Organization (CSIRO) Joint Grant (154144KYSB20150045) and partially supported by Guangzhou Municipal Industry and Research Collaborative Innovation Program (201508020248) and Guangzhou Municipal Clinical Medical Center Program (155700012) and the Key Project of Guangdong Provincial Department of Science and Technology (2013-137-115).

\section{Conflict of Interest}

The authors have no financial conflicts of interests.

\section{References}

[1] Jindani, A., Dore, C.J. and Mitchison, D.A. (2003) Bactericidal and Sterilizing Activities of Antituberculosis Drugs during the First 14 Days. American Journal of Respiratory and Critical Care Medicine, 167, 1348-1354. http://dx.doi.org/10.1164/rccm.200210-11250C

[2] Mitchison, D.A. (2000) Role of Individual Drugs in the Chemotherapy of Tuberculosis. International Journal of Tuberculosis and Lung Disease, 4, 796-806.

[3] Heifets, L. and Lindholmlevy, P. (1992) Pyrazinamide Sterilizing Activity in Vitro against Semidormant Mycobacterium-Tuberculosis Bacterial-Populations. American Review of Respiratory Disease, 145, 1223-1225. http://dx.doi.org/10.1164/ajrccm/145.5.1223

[4] East African-British Medical Research Councils (1972) Controlled Clinical Trial of Short-Course (6-Month) Regimens of Chemotherapy for Treatment of Pulmonary Tuberculosis. Lancet, 1, 1079-1085.

[5] Gumbo, T., Dona, C.S.W.S., Meek, C. and Leff, R. (2009) Pharmacokinetics-Pharmacodynamics of Pyrazinamide in a 
Novel in Vitro Model of Tuberculosis for Sterilizing Effect: A Paradigm for Faster Assessment of New Antituberculosis Drugs. Antimicrobial Agents and Chemotherapy, 53, 3197-3204. http://dx.doi.org/10.1128/AAC.01681-08

[6] Temple, B., Ayakaka, I., Ogwang, S., Nabanjja, H., Kayes, S., Nakubulwa, S., et al. (2008) Rate and Amplification of Drug Resistance among Previously-Treated Patients with Tuberculosis in Kampala, Uganda. Clinical Infectious Diseases, 47, 1126-1134. http://dx.doi.org/10.1086/592252

[7] Blumberg, H.M., Burman, W.J., Chaisson, R.E., Daley, C.L., Etkind, S.C., Friedman, L.N., et al. (2003) American Thoracic Society/Centers for Disease Control and Prevention/Infectious Diseases Society of America: Treatment of Tuberculosis. American Journal of Respiratory and Critical Care Medicine, 167, 603-662. http://dx.doi.org/10.1164/rccm.167.4.603

[8] Kubica, G.P., Cohn, M.L., Dye, W.E. and Middlebrook, G. (1963) Sputum Digestion and Decontamination with NAcetyl-L-Cysteine-Sodium Hydroxide for Culture of Mycobacteria. American Review of Respiratory Disease, 87, 775779.

[9] Basic Specialty Board in Chinese Antituberculosis Association (2006) Tuberculosis Diagnoiss Laboratory Testing Protocols. 1st Version, Chinese Education Culture Press, Beijing, 13-30.

[10] World Health Organisation (1998) Laboratory Services in Tuberculosis Control. World Health Organization, Geneva. http://apps.who.int/iris/bitstream/10665/65942/1/WHO_TB_98.258_\%28part1\%29.pdf

[11] Palomino, J.C., Traore, H., Fissette, K. and Portaels, F. (1999) Evaluation of Mycobacteria Growth Indicator Tube (MGIT) for Drug Susceptibility Testing of Mycobacterium tuberculosis. International Journal of Tuberculosis and Lung Disease, 3, 344-348.

[12] Cui, Z., Wang, J., Lu, J., Huang, X., Zheng, R. and Hu, Z. (2013) Evaluation of Methods for Testing the Susceptibility of Clinical Mycobacterium tuberculosis Isolates to Pyrazinamide. Journal of Clinical Microbiology, 51, 1374-1380. http://dx.doi.org/10.1128/JCM.03197-12

[13] World Health Organisation (2004) Compendium of Indicators for Monitoring and Evaluating National Tuberculosis Programs. World Health Organisation, Geneva. http://apps.who.int/iris/bitstream/10665/68768/1/WHO HTM TB 2004.344.pdf

[14] Singla, R., Osman, M.M., Khan, N., Al-Sharif, N., Al-Sayegh, M.O. and Shaikh, M.A. (2003) Factors Predicting Persistent Sputum Smear Positivity among Pulmonary Tuberculosis Patients 2 Months after Treatment. International Journal of Tuberculosis and Lung Disease, 7, 58-64.

[15] Salaniponi, F.M.L., Christensen, J.J., Gausi, F., Kwanjana, J.J. and Harries, A.D. (1999) Sputum Smear Status at Two Months and Subsequent Treatment Outcome in New Patients with Smear-Positive Pulmonary Tuberculosis. International Journal of Tuberculosis and Lung Disease, 3, 1047-1048.

[16] Fortun, J., Martin-Davila, P., Molina, A., Navas, E., Manuel Hermida, J., Cobo, J., et al. (2007) Sputum Conversion among Patients with Pulmonary Tuberculosis: Are There Implications for Removal of Respiratory Isolation? Journal of Antimicrobial Chemotherapy, 59, 794-798. http://dx.doi.org/10.1093/jac/dkm025

[17] Blower, S.M. and Chou, T. (2004) Modeling the Emergence of the "hot Zones": Tuberculosis and the Amplification Dynamics of Drug Resistance. Nature Medicine, 10, 1111-1116. http://dx.doi.org/10.1038/nm1102

[18] Zhang, Y. and Mitchison, D. (2003) The Curious Characteristics of Pyrazinamide: A Review. International Journal of Tuberculosis and Lung Disease, 7, 6-21.

[19] Ahmad, Z., Tyagi, S., Minkowski, A., Almeida, D., Nuermberger, E.L., Peck, K.M. et al. (2012) Activity of 5-chloropyrazinamide in Mice Infected with Mycobacterium tuberculosis or Mycobacterium bovis. Indian Journal of Medical Research, 136, 808-814.

[20] Lanoix, J.-P., Betoudji, F. and Nuermberger, E. (2014) Novel Regimens Identified in Mice for Treatment of Latent Tuberculosis Infection in Contacts of Patients with Multidrug-Resistant Tuberculosis. Antimicrobial Agents and Chemotherapy, 58, 2316-2321. http://dx.doi.org/10.1128/AAC.02658-13

[21] Mitchison, D.A. and Nunn, A.J. (1986) Influence of Initial-Drug Resistance on the Response to Short-Course Chemotherapy of Pulmonary Tuberculosis. American Review of Respiratory Disease, 133, 423-430.

[22] Zhang, Y., Chang, K.C., Leung, C.-C., Yew, W.W., Gicquel, B., Fallows, D., et al. (2012) “Z ${ }^{\mathrm{S}}$-MDR-TB” versus “ZR-MDR-TB”: Improving Treatment of MDR-TB by Identifying Pyrazinamide Susceptibility. Emerging Microbes \& Infections, 1, e5. http://dx.doi.org/10.1038/emi.2012.18

[23] Rao, Y., Tan, Y. and Tan, S. (2014) The Influence of Pyrazinamide Resistance on the Treatment Outcome of Initial Treatment of Culture Positive Patients. The Journal of Practical Medicine, 30, 127-129.

[24] Budzik, J.M., Jarlsberg, L.G., Higashi, J., Grinsdale, J., Hopewell, P.C., Kato-Maeda, M., et al. (2014) Pyrazinamide Resistance, Mycobacterium tuberculosis Lineage and Treatment Outcomes in San Francisco, California. PLoS ONE, 9, e95645. http://dx.doi.org/10.1371/journal.pone.0095645 
[25] Liang, B., He, Q., Huang, R., Wu, H., Ouyang, C., He, L., et al. (2013) Analysis of Smear Conversion of Smear Positive Tuberculosis Patients in the Retreatment Group at the End of Month 2 Chemotherapy. Chinese Journal of Antituberculosis, 35, 965-968.

[26] Zhang, T., Li, S.-Y. and Nuermberger, E.L. (2012) Autoluminescent Mycobacterium tuberculosis for Rapid, Real-Time, Non-Invasive Assessment of Drug and Vaccine Efficacy. PLoS ONE, 7, e29774. http://dx.doi.org/10.1371/journal.pone.0029774

[27] Prabhakar, R. (1986) A Controlled Clinical-Trial of 3-Month and 5-Month Regimens in the Treatment of Sputum-Positive Pulmonary Tuberculosis in South-India. American Review of Respiratory Disease, 134, 27-33.

[28] Zhang, T., Zhang, M., Rosenthal, I.M., Grosset, J.H. and Nuermberger, E.L. (2009) Short-Course Therapy with Daily Rifapentine in a Murine Model of Latent Tuberculosis Infection. American Journal of Respiratory and Critical Care Medicine, 180, 1151-1157. http://dx.doi.org/10.1164/rccm.200905-0795OC

[29] Monin, L. and Khader, S.A. (2014) Chemokines in Tuberculosis: The Good, the Bad and the Ugly. Seminars in Immunology, 26, 552-558. http://dx.doi.org/10.1016/j.smim.2014.09.004

[30] Manca, C., Koo, M.-S., Peixoto, B., Fallows, D., Kaplan, G. and Subbian, S. (2013) Host Targeted Activity of Pyrazinamide in Mycobacterium tuberculosis Infection. PLOS ONE, 8, e74082. http://dx.doi.org/10.1371/journal.pone.0074082

[31] Cardona, P.J. and Ruiz-Manzano, J. (2004) On the Nature of Mycobacterium tuberculosis-Latent Bacilli. European Respiratory Journal, 24, 1044-1051. http://dx.doi.org/10.1183/09031936.04.00072604

[32] Gengenbacher, M. and Kaufmann, S.H.E. (2012) Mycobacterium tuberculosis: Success through Dormancy. FEMS Microbiology Reviews, 36, 514-532. http://dx.doi.org/10.1111/j.1574-6976.2012.00331.x

[33] Wade, M.M. and Zhang, Y. (2004) Anaerobic Incubation Conditions Enhance Pyrazinamide Activity against Mycobacterium tuberculosis. Journal of Medical Microbiology, 53, 769-773. http://dx.doi.org/10.1099/jmm.0.45639-0

[34] Zhang, Y., Wade, M.M., Scorpio, A., Zhang, H. and Sun, Z.H. (2003) Mode of Action of Pyrazinamide: Disruption of Mycobacterium tuberculosis Membrane Transport and Energetics by Pyrazinoic Acid. Journal of Antimicrobial Chemotherapy, 52, 790-795. http://dx.doi.org/10.1093/jac/dkg446

[35] Yee, D.P., Menzies, D. and Brassard, P. (2012) Clinical Outcomes of Pyrazinamide-Monoresistant Mycobacterium tuberculosis in Quebec. International Journal of Tuberculosis and Lung Disease, 16, 604-609.

[36] Jo, K.-W., Yoo, J.-W., Hong, Y., Lee, J.S., Lee, S.-D., Kim, W.S., et al. (2014) Risk Factors for 1-Year Relapse of Pulmonary Tuberculosis Treated with a 6-Month Daily Regimen. Respiratory Medicine, 108, 654-659. http://dx.doi.org/10.1016/j.rmed.2014.01.010

[37] Tan, Y., Hu, Z., Zhang, T., Cai, X., Kuang, H., Liu, Y., et al. (2014) Role of pncA and rpsA Gene Sequencing in Detection of Pyrazinamide Resistance in Mycobacterium tuberculosis Isolates from Southern China. Journal of Clinical Microbiology, 52, 291-297. http://dx.doi.org/10.1128/JCM.01903-13

[38] Scorpio, A. and Zhang, Y. (1996) Mutations in pncA, a Gene Encoding Pyrazinamidase/Nicotinamidase, Cause Resistance to the Antituberculous Drug Pyrazinamide in Tubercle Bacillus. Nature Medicine, 2, 662-667. http://dx.doi.org/10.1038/nm0696-662

[39] Chinese Medical Association (2005) Clinical Diagnose and Treatment Instructions: Tuberculosis. People’s Medical Publishing House, Beijing, 109-110. 